\title{
Bruxismo en la infancia: ¿cómo tratar?
}

\author{
Arielle Figueredo Carnaúba de Araújo, ${ }^{1}$ \\ Giovanna Samara Barros Dorvillé, ${ }^{1}$ \\ Nayara Medeiros da Silva Guerra Sales, ${ }^{1}$ \\ Nicole Beatriz Barros de Sá Freitas, ${ }^{1}$ \\ Ana Lídia Soares Cota. ${ }^{2}$
}

\section{Resumen}

El bruxismo infantil es una condición definida por la acción de apretar o rechinar los dientes involuntariamente, siendo así de etiologia multifactorial. Cuando no es tratado tempranamente, puede causar daño al sistema estomatognático, causando cambios orofaciales. De esta forma, este artículo tiene como objetivo abordar las diferentes opciones de tratamiento para el bruxismo en niños. Esta es una revisión integradora realizada a través de las bases de datos SciELO y PubMed, la cual incluye un total de 14 artículos, respetando los criterios de inclusión y exclusión. En general, el tratamiento debe ser cumplido realizado de manera multidisciplinaria, incluyendo la incorporación del cirujano dentista, doctor, psicólogo, fisioterapeuta y logopeda para mejorar la calidad de vida del individuo. A través de la exclusión de los posibles factores que determinan la enfermedad, tiene como objetivo promover la reducción de la actividad parafuncional. Para lograr eso, podemos usar placas oclusales, medicamentos como analgésicos, antiinflamatorio, benzodiacepinas, relajantes musculares, además de las hierbas medicinales, así como también, terapia de masaje, criolipólisis, acupuntura, entre otros. Sin embargo, mas estudios son necesarios para probar la efectividad de estas terapias. Se puede concluir que hasta el momento presente, lo que se puede ofrecer es un tratamiento paliativo y multidisciplinario que permite la reducción de complicaciones patológicas, favoreciendo así, la salud del niño.

Palabras clave: Niño, bruxismo y tratamiento. 
Artigo da revisao

\section{Bruxismo na infância: como tratar?}

\section{Resumo}

O bruxismo infantil é uma condição definida pela ação de apertar ou ranger os dentes de forma involuntária sendo de etiologia multifatorial. Quando não interferida precocemente pode gerar danos ao sistema estomatognático, ocasionando alterações orofaciais. Dessa forma, o presente artigo tem como objetivo abordar as diferentes opções de tratamento para o bruxismo em crianças. Trata-se de uma revisão integrativa realizada por meio das bases de dados SciELO e PubMed, foram incluídos um total de 14 artigos, respeitando os critérios de inclusão e exclusão. De forma geral, o tratamento deve ser realizado multidisciplinarmente, englobando a adição do cirurgiãodentista, médico, psicólogo, fisioterapeuta e fonoaudiólogo, com intuito de proporcionar melhora da qualidade de vida ao indivíduo. Através da exclusão dos possíveis fatores determinantes da doença, visa-se a promoção da redução da atividade parafuncional. Para isso, pode-se lançar mão de placas oclusais, medicamentos, como analgésicos, anti-inflamatórios, benzodiazepínicos, relaxantes musculares, além de fitoterápicos, bem como, massagem terapêutica, criolipólise, acupuntura, entre outros. Contudo, é necessário que mais estudos comprovem a eficácia dos referidos terapêuticos. Assim sendo, conclui-se que até o presente momento, o que se pode oferecer é um tratamento paliativo e multidisciplinar que possibilite a diminuição das complicações patológicas e desta forma favorecendo o estado de saúde da criança.

Palavras chaves: Criança, bruxismo e tratamento.

Article of revision

\section{Bruxism in childhood: how to treat?}

\section{Abstract}

The bruxism in the childhood is a multifactorial condition defined by the act of involuntarily clenching or grinding the teeth. When not interfered early can cause damage to the stomatognathic system, causing orofacial changes. Thus, this article aims to address the different treatment options for bruxism in children, this is an integrative review conducted through the SciELO and PubMed databases, a total of 14 articles were included, respecting the inclusion and exclusion criteria. In general, the treatment should be performed in a multidisciplinary manner, including the addition of the dental surgeon, physician, psychologist, physiotherapist and speech therapist, in order to improve the quality of life of the individual. By excluding possible determinants of the disease, the aim is to promote the reduction of parafunctional activity. For this, it can use occlusal plaques, medications such as analgesics, antiinflammatory drugs, benzodiazepines, muscle relaxants, as well as herbal medicines, as well as massage therapy, cryolipolysis, acupuncture, among 
others. However, further studies need to prove the effectiveness of such therapies. Therefore, it can be concluded that, until now, what can be offered is a palliative and multidisciplinary treatment that

\section{Introducción}

Caracterizado por la acción de rechinar o apretar los dientes, el bruxismo es considerado un hábito no funcional e involuntario del sistema estomatognático. Tiene mayor ocurrencia durante el sueño y aunque ésta condición es más frecuente en adultos, también puede estar presente desde la infancia afectando del $40 \%$ al $49,6 \%$ de los niños de 3 a 6 años. ${ }^{1,2,3}$

La etiologia del bruxismo es multifactorial y compleja, relacionándose a una diversidad de causas, como factores dentales representados por la oclusión, posición dental, movilidad, patrón de movimiento mandibular, morfologia y restauraciones iatrogénicas. La investigación ha mencionado la influencia de factores psicoemocionales, por someter al individuo a la tensión, estrés, miedo, rabia y ansiedad, culminando en la descarga de toda la agresividad y en los componentes del sistema estomatognático. ${ }^{1,4,5}$

Algunas condiciones sistémicas como los cambios en el tracto digestivo y nutricional, el desequilíbrio endocrino, los trastornos del sueño, alergias y los factores hereditarios también se han enumerado como factores etiológicos. Sin embargo, el consumo de alimentos xantanos (café, té, chocolate, refrescos tipo cola), anfetaminas, álcohol y tabaco necesitan ser evaluados, una vez que, estimulando el sistema nervioso central aumenta la ansiedad y estrés de las personas. ${ }^{4,5}$ enables the reduction of pathological complications and thus favoring the child's health status.

Key words: Children, bruxism and treatment.

Dependiendo del grado de gravedad el bruxismo puede causar varias consecuencias para la cavidad bucal, como el desgaste dental, localizado en la región incisal de los dientes anteriores, aumento de la sensibilidad dental, dolores musculares en la cara y cambios en la articulación temporomandibular, que se convierten en un problema debido al crecimiento óseo incompleto del niño. ${ }^{1,2,6}$

Este hábito parafuncional puede manifestarse durante el sueño, siendo así denominado de bruxismo nocturno y durante el dia conocido por el bruxismo diurno. Su principal diferencia recae sobre el estado de conciencia en el cual el paciente se encuentra durante la ejecución de los movimientos, es decir, una acción inconsciente $\mathrm{O}$ una actividad semivoluntaria, respectivamente. ${ }^{3,6}$ Además de la clasificación, el bruxismo es considerado primario, cuando el individuo no tiene una causa sistémica o psíquica evidente y secundario cuando está asociado con factores iatrogénicos o trastornos clínicos y/o neurológicos. ${ }^{7}$

Con el avance de la investigación sobre el tratamiento del buxismo, se hace evidente una gran dificuldad clínica al buscar y reconocer los factores etiológicos asociados. Esto representa un desafío en la aplicación de medidas terapéuticas efectivas, generando aprensión entre profesionales y familiares, debido al potencial para tener un impacto negativo en la calidad de vida de los afectados. ${ }^{6,8}$ Dado lo anterior, 
el objetivo del presente trabajo es discutir las principales formas del tratamiento indicado para el bruxismo en los niños.

\section{Materiales y métodos}

El artículo trata de una revisión integradora de literatura, que consiste en utilizar una técnica metodológica que incluye el análisis de investigaciones que sirven como base para la tomada de decisiones y la mejora de la práctica clínica, permitiendo la producción del estado de conocimientos sobre un tema determinado, además de señalar lagunas de conocimientos que necesitan ser completadas con la realización de nuevos estudios. ${ }^{9}$

En el presente estudio, la formulación del tema surgió de la pregunta guía: ¿cómo tratar el bruxismo en los niños?

Las bases de datos utilizadas fueron la Scientific Electronic Library Online (SciELO), considerando como descriptores: niño; Bruxismo y Tratamiento; y la

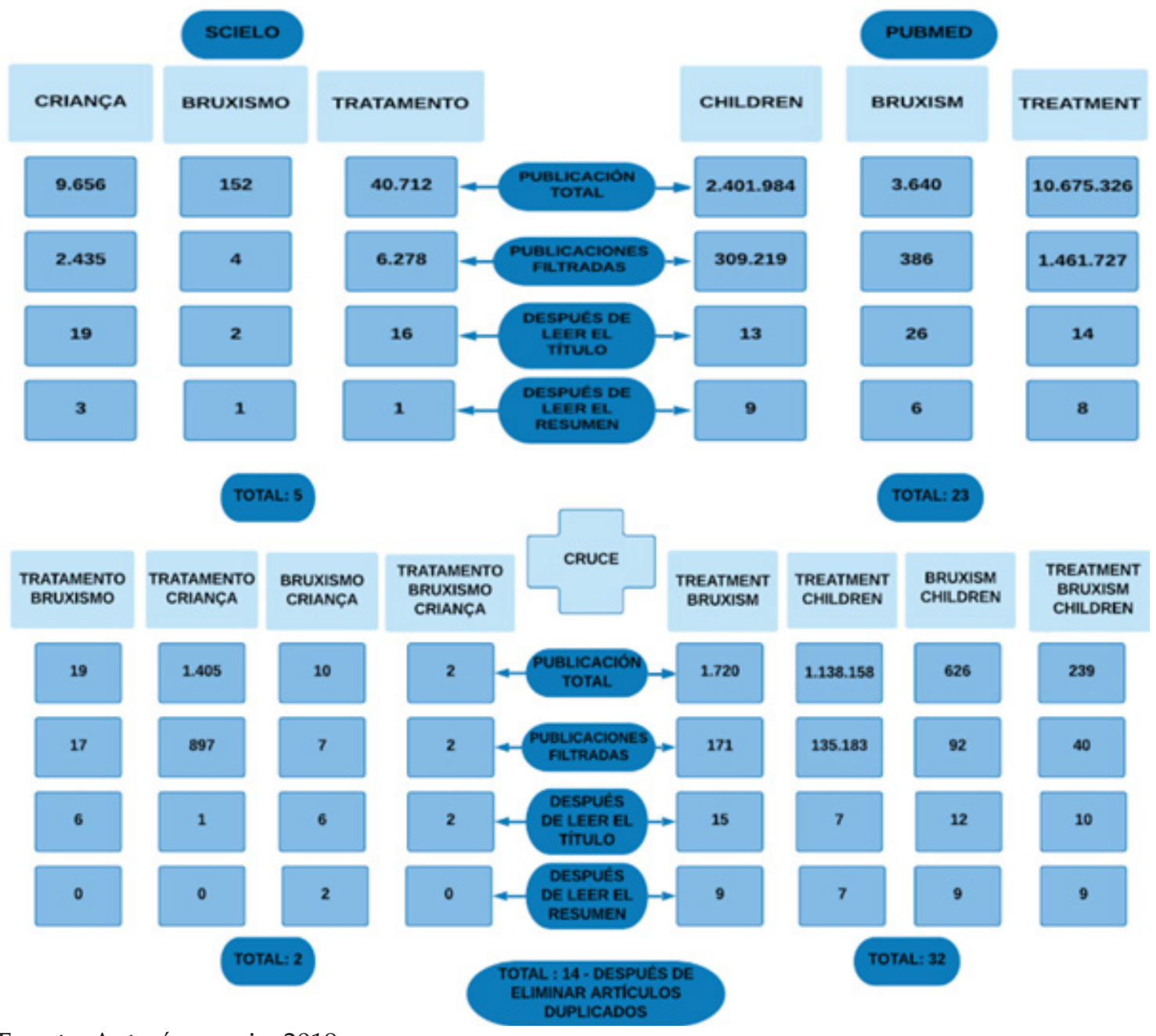

Fuente: Autoría propia, 2019.

Figura 1. Diagrama de flujo con publicaciones encontradas entre los años 2009 a 2019 segundo las bases de datos SciELO y PubMed 
plataforma U.S. National Library of Medicine (PubMed), utilizando los descriptores, Children; Bruxism e Treatment. Primero, se realizó la búsqueda de artículos a través de los descriptores individuales y, en seguida, las intersecciones de los descritores utilizando el operador boleano and.

Los criterios de inclusión para la selección de la muestra fueron: artículos publicados en portugués e inglés, totalmente disponibles publicados entre
2009 y 2019, y después de leer el título y el resumen, se aborda la temática del estudio. Libros, capítulos de libro, trabajos de terminación de curso, disertaciones y tesis fueron excluidos del estudio (Figura 1).

Después de leer los resúmenes, 62 artículos fueron seleccionados por obedecer a los criterios de inclusión. Sin embargo, solo se seleccionaron 14 artículos debido a la duplicidad (Tabla 1).

Tabla 1. Presentación de las características de los artículos incluidos en la Revisión Integrativa del SciELO y PubMed.

\begin{tabular}{|c|c|c|c|c|}
\hline TÍTULO & AUTORES/AÑO & OBJETIVO & $\begin{array}{l}\text { RESULTADOS } \\
\text { PRINCIPALES }\end{array}$ & CONCLUSIÓN \\
\hline $\begin{array}{l}\text { Bruxismo infantil: } \\
\text { Una señal de adver- } \\
\text { tencia para dentista } \\
\text { pediátricos y pedia- } \\
\text { tras. }\end{array}$ & $\begin{array}{l}\text { Diniz MB, Silva RC, } \\
\text { Zuano ACC. } 2009 .\end{array}$ & $\begin{array}{l}\text { Presentar una revi- } \\
\text { sión de la literatura } \\
\text { sobre bruxismo en la } \\
\text { infancia, presentando } \\
\text { reales características, } \\
\text { señales, sintomas y } \\
\text { factores etiológicos. }\end{array}$ & $\begin{array}{l}\text { La investigación } \\
\text { destaca la importan- } \\
\text { cia de un tratamiento } \\
\text { multidisciplinar para } \\
\text { el bruxismo infantil } \\
\text { debido a su etiología } \\
\text { multifactorial. }\end{array}$ & $\begin{array}{l}\text { El tratamiento para } \\
\text { el bruxismo debe } \\
\text { ocorrir de forma mul- } \\
\text { tidisciplinar, abran- } \\
\text { gendo pediatra,den- } \\
\text { tista pediátrico y } \\
\text { psicologo, apuntando } \\
\text { al desarrollo integral } \\
\text { del niño. }\end{array}$ \\
\hline $\begin{array}{l}\text { Bruxismo del sueño: } \\
\text { posibilidades tera- } \\
\text { péuticas basadas en } \\
\text { las evidencias. }\end{array}$ & $\begin{array}{l}\text { Machado E, Machado } \\
\text { P, Cunali PA, Fabbro } \\
\text { CD. } 2011 .\end{array}$ & $\begin{array}{l}\text { Analizar y discutir } \\
\text { maneras de tra- } \\
\text { tamiento para el } \\
\text { bruxismo infantil con } \\
\text { base en una revisión } \\
\text { sistemática. }\end{array}$ & $\begin{array}{l}\text { Es posible encontrar } \\
\text { una variedad de } \\
\text { opciones terapéuticas } \\
\text { para el tratamiento } \\
\text { del bruxismo en la } \\
\text { infancia. }\end{array}$ & $\begin{array}{l}\text { En su mayoría, } \\
\text { las opciones tera- } \\
\text { péuticas no tienen } \\
\text { bases científicas que } \\
\text { los sostienen y/o } \\
\text { comproben que las } \\
\text { terapias invasivas son } \\
\text { superiores a las tera- } \\
\text { pias no-invasivas. }\end{array}$ \\
\hline $\begin{array}{l}\text { Effect of occlusal } \\
\text { splints on the tem- } \\
\text { poromandibular di- } \\
\text { sorders, dental wear } \\
\text { and anxiety of bruxist } \\
\text { children. } \\
\text { (Efecto de las placas } \\
\text { oclusales en los tras- } \\
\text { tornos temporoman- } \\
\text { dibulares, desgaste } \\
\text { dental y ansiedad en } \\
\text { los niños con bruxis- } \\
\text { mo). }\end{array}$ & $\begin{array}{l}\text { Restrepo CC, Medina } \\
\text { I, Patiño I. } 2011 .\end{array}$ & $\begin{array}{l}\text { Avaluar la efectivi- } \\
\text { dad de las placas } \\
\text { oclusales para reducir } \\
\text { los señales y sínto- } \\
\text { mas del bruxismo, } \\
\text { como los trastornos } \\
\text { temporomandibula- } \\
\text { res, desgaste dental } \\
\text { y ansiedad en los } \\
\text { niños. }\end{array}$ & $\begin{array}{l}\text { El grupo experi- } \\
\text { mental con niños } \\
\text { de } 40,3 \text { meses nos } \\
\text { mostraron diferencias } \\
\text { en relación con los } \\
\text { niveles de ansiedad } \\
\text { y desgaste dental, en } \\
\text { comparación con el } \\
\text { grupo de control. }\end{array}$ & $\begin{array}{l}\text { El uso de placas de } \\
\text { mordida rígidas } \\
\text { oclusales no fue } \\
\text { eficaz para reducir las } \\
\text { señales del bruxismo, } \\
\text { en su conjunto, pero } \\
\text { si redujo la desvia- } \\
\text { ción mandibular en la } \\
\text { apertura de la boca. }\end{array}$ \\
\hline
\end{tabular}




\begin{tabular}{|c|c|c|c|c|}
\hline TÍTULO & AUTORES/AÑO & $\overline{\text { OBJETIVO }}$ & $\begin{array}{l}\text { RESULTADOS } \\
\text { PRINCIPALES }\end{array}$ & CONCLUSIÓN \\
\hline $\begin{array}{l}\text { Buspirone use in the } \\
\text { treatment of ato- } \\
\text { moxetine-induced } \\
\text { bruxism. } \\
\text { (Uso de buspirona } \\
\text { en el tratamiento del } \\
\text { bruxismo inducido } \\
\text { por atomoxetina). }\end{array}$ & $\begin{array}{l}\text { Yuce M, Karabekirog, } \\
\text { KSG, Mujdeci M; } \\
\text { Oran M. } 2013 .\end{array}$ & $\begin{array}{l}\text { Relatar la aparición } \\
\text { del bruxismo relacio- } \\
\text { nado con el uso de la } \\
\text { droga medicamento } \\
\text { atomoxetina, y el tra- } \\
\text { tamiento de bruxismo } \\
\text { usando buspirona. }\end{array}$ & $\begin{array}{l}\text { El buspirona revela } \\
\text { una eficacia en el } \\
\text { tratamiento del bru- } \\
\text { xismo inducido por } \\
\text { atomoxetina. }\end{array}$ & $\begin{array}{l}\text { Al prescribir ato- } \\
\text { moxetina debe } \\
\text { recordarse que puede } \\
\text { estar predisponien- } \\
\text { do al bruxismo, y la } \\
\text { presencia de bruxis- } \\
\text { mo debe cuestionarse } \\
\text { durante las visitas } \\
\text { psiquiátricas. }\end{array}$ \\
\hline $\begin{array}{l}\text { Treatment of bruxism } \\
\text { with hydroxyzine. } \\
\text { (Tratamiento de bru- } \\
\text { xismo con hidroxi- } \\
\text { cina). }\end{array}$ & Gahnizade A. 2013. & $\begin{array}{l}\text { Relatar el tratamiento } \\
\text { de bruxismo del sue- } \\
\text { ño con hidroxicina en } \\
\text { tres niños, acompaña- } \\
\text { das por } 2 \text { meses. }\end{array}$ & $\begin{array}{l}\text { Los padres informa- } \\
\text { ron como resultado } \\
\text { una reducción signifi- } \\
\text { cativa en pontuación } \\
\text { de la Escala Visual } \\
\text { Analógica (EVA) } \\
\text { después de tomar } \\
\text { hidroxicina por un } \\
\text { mês. }\end{array}$ & $\begin{array}{l}\text { La hidroxicina puede } \\
\text { ser eficaz en el trata- } \\
\text { miento del bruxismo } \\
\text { en niños, resultando } \\
\text { beneficioso realizar } \\
\text { estudios controlados } \\
\text { por placebo. }\end{array}$ \\
\hline $\begin{array}{l}\text { Evaluation of Cra- } \\
\text { nio-cervical Posture } \\
\text { in Children with Bru- } \\
\text { xism Before and After } \\
\text { Bite Plate Therapy: A } \\
\text { Pilot Project. } \\
\text { (Evaluación de la } \\
\text { postura craneocer- } \\
\text { vical en niños con } \\
\text { bruxismo antes y de- } \\
\text { pués de la terapia con } \\
\text { placa de mordida: un } \\
\text { proyecto piloto). }\end{array}$ & $\begin{array}{l}\text { Bortolleto CC, Silva } \\
\text { FC, Silva PFC, Go- } \\
\text { doy, CHL; Albertini } \\
\text { R. et. al. } 2014 .\end{array}$ & $\begin{array}{l}\text { Avaluar el efecto de } \\
\text { una placa de mordida } \\
\text { en la postura craneo- } \\
\text { cervical de niños con } \\
\text { bruxismo. }\end{array}$ & $\begin{array}{l}\text { No se encontraron } \\
\text { resultados significa- } \\
\text { tivos en la postura } \\
\text { craneocervical de los } \\
\text { niños en un mes de } \\
\text { uso de la placa de } \\
\text { mordida. }\end{array}$ & $\begin{array}{l}\text { Puede ocurrir apenas } \\
\text { una reducción en el } \\
\text { ângulo craneo cervi- } \\
\text { cal cuando la placa } \\
\text { de mordida permane- } \\
\text { ce en posición. }\end{array}$ \\
\hline $\begin{array}{l}\text { The effect of hy- } \\
\text { droxyzine on treating } \\
\text { bruxism of 2- to } \\
\text { 14-year-old children } \\
\text { admitted to the clinic } \\
\text { of Bandar Abbas } \\
\text { Children Hospital in } \\
\text { 2013-2014. } \\
\text { (El efecto de la } \\
\text { hidroxicina en el } \\
\text { tratamiento del bru- } \\
\text { xismo de niños de } 2 \text { a } \\
14 \text { años admitidas en } \\
\text { la clínica de Hospital } \\
\text { Infantil Bandar Abbas } \\
\text { em 2013-2014). }\end{array}$ & $\begin{array}{l}\text { Rahmati M, Moa- } \\
\text { yediA, Shahvari } \\
\text { SZ, Golmirzaei J, } \\
\text { Zahirinea M, Abbasi } \\
\text { B. } 2015 \text {. }\end{array}$ & $\begin{array}{l}\text { Estudiar el efecto de } \\
\text { la hidroxicina en el } \\
\text { tratamiento del bru- } \\
\text { xismo de los niños de } \\
2 \text { a } 14 \text { años de edad. }\end{array}$ & $\begin{array}{l}\text { La prescripción de } \\
\text { hidroxicina por } 4 \\
\text { semanas tuvo un } \\
\text { efecto considerable } \\
\text { en la disminuición } \\
\text { de la severidad del } \\
\text { bruxismo entre los } \\
\text { grupos evaluados. }\end{array}$ & $\begin{array}{l}\text { Es necessario tener } \\
\text { en cuenta los efectos } \\
\text { secundarios de la hi- } \\
\text { droxicina para tomar } \\
\text { decisiones de trata- } \\
\text { miento adecuadas. }\end{array}$ \\
\hline $\begin{array}{l}\text { Clinical management } \\
\text { of childhood bru- } \\
\text { xism. } \\
\text { (Manejo clínico del } \\
\text { bruxismo infantil) }\end{array}$ & $\begin{array}{l}\text { Alfaya, TA, Tannure, } \\
\text { PN, Barcelos R; Dip } \\
\text { EC,; Uemoto L, Gou- } \\
\text { vêa, CVD. } 2015 \text {. }\end{array}$ & $\begin{array}{l}\text { Relataron un caso } \\
\text { clínico de un paciente } \\
\text { del sexo masculino } \\
\text { de nueve años de } \\
\text { edad, presentando } \\
\text { quejas de dolor de } \\
\text { cabeza y contracción } \\
\text { dental durante la } \\
\text { vigilia. }\end{array}$ & $\begin{array}{l}\text { Hubo una disminu- } \\
\text { ción en el dolor de } \\
\text { cabeza después de un } \\
\text { mes de uso de placa } \\
\text { miorelajante y del } \\
\text { seguimiento dental } \\
\text { y psicológico que } \\
\text { todavia se realiza. }\end{array}$ & $\begin{array}{l}\text { La placa miorrelajan- } \\
\text { te es una gran opción } \\
\text { terapéutica para estos } \\
\text { casos de bruxismo,sin } \\
\text { embargo también es } \\
\text { necesario explorar } \\
\text { otras áreas en el enfo- } \\
\text { que terapéutico como } \\
\text { el psicologo. }\end{array}$ \\
\hline
\end{tabular}




\begin{tabular}{|c|c|c|c|c|}
\hline TÍTULO & AUTORES/AÑO & OBJETIVO & $\begin{array}{l}\text { RESULTADOS } \\
\text { PRINCIPALES }\end{array}$ & CONCLUSIÓN \\
\hline $\begin{array}{l}\text { Evaluation of elec- } \\
\text { tromyographic sig- } \\
\text { nals in children with } \\
\text { bruxism before and } \\
\text { after therapy with } \\
\text { Melissa Officinalis L-a } \\
\text { randomized contro- } \\
\text { lled clinical trial. } \\
\text { (Evaluación de } \\
\text { señales electromio- } \\
\text { gráficos en niños con } \\
\text { bruxismo antes y } \\
\text { después de la terapia } \\
\text { con Melissa Officinalis } \\
\text { L - un ensayo clínico } \\
\text { aleatorizado y contro- } \\
\text { lado). }\end{array}$ & $\begin{array}{l}\text { Bortolleto CC, Silva } \\
\text { FC, Salgueiro MCC, } \\
\text { Motta LJ, Curiki LM. } \\
2016 .\end{array}$ & $\begin{array}{l}\text { Avaluar las señales } \\
\text { electromiográficas en } \\
\text { el músculo temporal } \\
\text { utilizando la prueba } \\
\text { BiteStrip en niños con } \\
\text { bruxismo del sueño } \\
\text { antes y después de } \\
\text { la terapia con Melissa } \\
\text { officinalis L. }\end{array}$ & $\begin{array}{l}\text { No fueron encontra- } \\
\text { das diferencias entre } \\
\text { la actividad muscular } \\
\text { inicial y final entre el } \\
\text { grupo } 1 \text { que ingirió } \\
\text { Melissa Officinalis y el } \\
\text { grupo } 2 \text { que recebió } \\
\text { un placebo. }\end{array}$ & $\begin{array}{l}\text { Se necesitan más } \\
\text { estudios para pro- } \\
\text { porcionar evidencia } \\
\text { científica sobre la } \\
\text { efectividad de Me- } \\
\text { lissa officinalis, Para } \\
\text { convertirse en una } \\
\text { alternativa asesible } \\
\text { en los servicios de } \\
\text { salud públicas para la } \\
\text { prevención y el trata- } \\
\text { miento de problemas } \\
\text { de salud bucal. }\end{array}$ \\
\hline $\begin{array}{l}\text { Evaluation of muscle } \\
\text { activity, bite force } \\
\text { and salivary corti- } \\
\text { sol in children with } \\
\text { bruxism before and } \\
\text { after low level laser } \\
\text { applied to acupoints: } \\
\text { study protocol for a } \\
\text { randomised contro- } \\
\text { lled trial. } \\
\text { (Evaluación de la ac- } \\
\text { tividad, la fuerza de } \\
\text { mordida y el cortisol } \\
\text { salival en niños con } \\
\text { bruxismo antes y } \\
\text { después del láser baja } \\
\text { potencia aplicada a } \\
\text { los puntos de acu- } \\
\text { puntura: protocolo } \\
\text { de estudio para un } \\
\text { estudio controlado } \\
\text { aleatorio). }\end{array}$ & $\begin{array}{l}\text { Salgueiro MCC, } \\
\text { Bortolleto CC, Horlia- } \\
\text { naACRT, Mota ACC, } \\
\text { Motta LJ, Motta, PB. } \\
\text { et al, } 2017 .\end{array}$ & $\begin{array}{l}\text { Avaluar los cam- } \\
\text { bios en la actividad } \\
\text { muscular, la fuerza } \\
\text { de la mordida y el } \\
\text { cortisol salival en los } \\
\text { niños con bruxismo } \\
\text { después la aplica- } \\
\text { ción de láser de baja } \\
\text { intensidad en puntos } \\
\text { de acupuntura. }\end{array}$ & $\begin{array}{l}\text { Los puntos: IG-4 } \\
\text { (Hegu), F-3 (Tai- } \\
\text { chong) y BP-6 } \\
\text { (Sanyinjiao), funciona } \\
\text { directamente para ali- } \\
\text { viar y calmar la ansie- } \\
\text { dad, disminuyendo la } \\
\text { contracción muscular } \\
\text { que a su vez, reduce } \\
\text { el bruxismo. }\end{array}$ & $\begin{array}{l}\text { La terapia a láser de } \\
\text { baja intensidad sobre } \\
\text { puntos de acupuntu- } \\
\text { ra es indicada en ni- } \\
\text { ños con bruxismo,sin } \\
\text { embargo, los efectos } \\
\text { (analgésico, disminu- } \\
\text { ción de los níveles de } \\
\text { cortisol,dolor y an- } \\
\text { siedad, por ejemplo) } \\
\text { de esta modalidad de } \\
\text { tratamiento aún no se } \\
\text { han estudiado. }\end{array}$ \\
\hline $\begin{array}{l}\text { Homeopathic therapy } \\
\text { for sleep bruxism in } \\
\text { a child: Findings of a } \\
\text { 2-year case report. }\end{array}$ & $\begin{array}{l}\text { Silva, CT; Guimarães } \\
\text { Primo L, Mangabeira } \\
\text { A; Maia LC, Fonse- } \\
\text { ca-Gonçalves A. } 2017 .\end{array}$ & $\begin{array}{l}\text { Informar sobre el uso } \\
\text { de la terapia ho- } \\
\text { meopática para el tra- } \\
\text { tamiento de bruxismo } \\
\text { en una paciente de } 8 \\
\text { años de edad. }\end{array}$ & $\begin{array}{l}\text { Después de un perío- } \\
\text { do de dos meses de } \\
\text { terapia homeopática } \\
\text { combinada (Phyto- } \\
\text { lacca decandra 12c y } \\
\text { Melissa officinalis 12c), } \\
\text { el bruxismo desa- } \\
\text { pareció y no mostró } \\
\text { recurrencia. }\end{array}$ & $\begin{array}{l}\text { El uso de la terapia } \\
\text { homeopática ha } \\
\text { tenido éxito y debe } \\
\text { ser visto como una } \\
\text { alternativa para tratar } \\
\text { el bruxismo en los } \\
\text { niños. }\end{array}$ \\
\hline $\begin{array}{l}\text { Bruxism in children: } \\
\text { Use of the Functio- } \\
\text { nal Plane of Monaco } \\
\text { (FPM). } \\
\text { (Bruxismo en niños: } \\
\text { uso do plan funcional } \\
\text { de Mónaco FPM). }\end{array}$ & $\begin{array}{l}\text { Ortu E, Pietropaoli D, } \\
\text { Marchetti E, Marchili } \\
\text { N, Marzo G, Monaco } \\
\text { A. } 2018 .\end{array}$ & $\begin{array}{l}\text { Informar el trata- } \\
\text { miento realizado en } \\
\text { un paciente de } 15 \\
\text { años del sexo mascu- } \\
\text { lino con bruxismo. }\end{array}$ & $\begin{array}{l}\text { Los autores utilizaron } \\
\text { solo el Plan Fun- } \\
\text { cional de Mónaco } \\
\text { (FPM), que se utilizó } \\
\text { durante el día. }\end{array}$ & $\begin{array}{l}\text { El ortodoncista trató } \\
\text { con éxito un caso de } \\
\text { ortopedia/ortodoncia } \\
\text { con el dispositivo } \\
\text { FPM. }\end{array}$ \\
\hline
\end{tabular}




\begin{tabular}{|c|c|c|c|c|}
\hline TÍTULO & AUTORES/AÑO & OBJETIVO & $\begin{array}{l}\text { RESULTADOS } \\
\text { PRINCIPALES }\end{array}$ & CONCLUSIÓN \\
\hline $\begin{array}{l}\text { Treatments of sleep } \\
\text { bruxism in children: } \\
\text { A systematic review } \\
\text { and meta-analysis. }\end{array}$ & $\begin{array}{l}\text { Lerardo G, Mazur M, } \\
\text { Luzzi V, Calcagnile F, } \\
\text { Ottolenghi L, Polime- } \\
\text { ni A. } 2018\end{array}$ & $\begin{array}{l}\text { Desarrollar un tra- } \\
\text { tamiento basado en } \\
\text { la evidencia para el } \\
\text { bruxismo en niños. }\end{array}$ & $\begin{array}{l}\text { El uso de la Hidroxi- } \\
\text { cina como terapia } \\
\text { mostró tener mayor } \\
\text { efectividad en el tra- } \\
\text { tamiento del bruxis- } \\
\text { mo del sueño }\end{array}$ & $\begin{array}{l}\text { Es necesario más } \\
\text { estudios con mayor } \\
\text { numero de niños, sin } \\
\text { embargo, con poca } \\
\text { evidencia, se cree en } \\
\text { la efectividad de la } \\
\text { hidroxicina. }\end{array}$ \\
\hline $\begin{array}{l}\text { The efficacy of low } \\
\text { and moderate dosage } \\
\text { of diazepam on sleep } \\
\text { bruxism in children: } \\
\text { A randomized place- } \\
\text { bo-controlled clinical } \\
\text { trial. } \\
\text { (La eficacia de la } \\
\text { dosis baja y modera- } \\
\text { da de diazepam en el } \\
\text { bruxismo del sueño } \\
\text { en niños: un ensayo } \\
\text { clínico aleatoriza- } \\
\text { do, controlado con } \\
\text { placebo). }\end{array}$ & $\begin{array}{l}\text { Mostafavi SN, Jafari } \\
\text { A, Hoseini SG, Kha- } \\
\text { demian M, Kelishadi } \\
\text { R. } 2019\end{array}$ & $\begin{array}{l}\text { Avaluar el efecto } \\
\text { del diazepam en el } \\
\text { control del bruxismo } \\
\text { en niños. }\end{array}$ & $\begin{array}{l}\text { Se observó que la gra- } \\
\text { vedad del bruxismo } \\
\text { disminuyó signifi- } \\
\text { cativamente en los } \\
3 \text { grupos(dosis baja } \\
\text { y placebo) durante } \\
2 \text { semanas, pero sin } \\
\text { diferencias entre los } \\
\text { grupos. }\end{array}$ & $\begin{array}{l}\text { El uso a corto plazo } \\
\text { de diazepam no fue } \\
\text { más efectivo que el } \\
\text { placebo en el trata- } \\
\text { miento del bruxismo } \\
\text { infantil. }\end{array}$ \\
\hline
\end{tabular}

\section{Discusión}

Uno de los métodos más confiables para diagnosticar el bruxismo infantil es el informe de los padres o tutores; sin embargo, debido al hecho de que los niños no siempre duermen cerca de los adultos, estos pueden no estar al tanto de los episodios relacionados con el crujir de dientes. Por lo tanto, el examen estándar para el diagnóstico de bruxismo es la polisomnografía. Sin embargo, la necesidad del paciente de dormir en el laboratorio hace que el procedimiento sea costoso y complejo para los niños. ${ }^{10,11,12}$

En este sentido, las estrategias sobre la efectividad del tratamiento del bruxismo infantil siguen siendo controvertidas, ya que que este trastorno es de carácter multifactorial, y requiere tratamiento multidisciplinario. Tanto el cirujano dentista, el odontólogo pediatra como los responsables, necesitan saber que tratamento por si solo puede no ser suficiente para tratar el bruxismo de su hijo. Por lo tanto, es importante que el profesional no descuide el tratamiento del paciente, siempre buscando las diferentes áreas que contemplan un tratamiento específico, cuando es indicado. ${ }^{12,13,14}$

Una de las formas terapéuticas más indicadas en odontología es la placa de mordida, que tiene como objetivo reducir la fricción y las cargas entre los dientes y estabilizar la articulación temporomandibular y la actividad neuromuscular. ${ }^{10,11,15}$ Sin embargo, Bortoletto et al. (2014), ${ }^{10}$ en un estudio observacional piloto, es decir, salvaguardando posibles limitaciones metodológicas, observaron que después de la remoción de la placa oclusal rígida la posición de la cabeza no se mantuvo y la carga no se eliminó pero, cuando 
se usa una placa de resina acrílica termoplástica, también conocida como placa miorrelajante, en un paciente con sobrecarga severa, Alfaya et al. (2015) ${ }^{15}$ detectaron una reducción en la queja principal del paciente (dolor de cabeza), pero sin eliminar el trastorno. Vale la pena mencionar que la placa miorrelajante recibió ajustes oclusales cada quince días, sin dañar la erupción de la dentición permanente y crecimiento óseo en niños.

A diferencia del reconocimiento en la literatura sobre drogas y tratamiento psicológico, los medicamentos homeopáticos no tienen evidencia científica, sin embargo, son sustancias naturales que se han descrito en algunos estudios como opciones para el alivio y la mejora de la salud general. Además, su uso en niños es más seguro, más accesible y sostenible, debido al hecho de ser controlado por el tiempo que es necesario, a diferencia de ciertos medicamentos que pueden generar algún efecto secundario. ${ }^{16}$

Silva et al. (2017), ${ }^{16}$ reportan que medicamentos recetados y descritos como fitoquímicos a base de hierbas naturales la Phytolacca decandra 12c como terapia para el bruxismo del sueño en un niño de 8 años del sexo masculino, cerca de la hora de acostarse tuvo como resultado una reducción de la trituración, pero debido al aumento de las pesadillas, la prescripción del medicamento Melissa officinalis 12 c fue indicado con lo cual se logró que la parasomnias y morderse las uñas, desparecieran por completo, permitiendo un sueño de calidad..

A través de una revisión sistemática y metanálisis, Lerardo et al. (2018) $)^{17}$ desarrollaron recomendaciones a través de la evidencia sobre la terapia para el bruxismo del sueño. Como resultado, los autores señalan que la hidroxicina es la opción más efectiva. Además, el uso de flurazepam y Melissa Officinalis también ha reducido los síntomas del bruxismo del sueño. Sin embargo, se concluyó que se necesitan más investigaciones basadas en el tratamiento y los criterios de diagnósticos estandarizados.

En algunas situaciones, puede haber una necesidad de tratamientos farmacológicos como analgésicos, antiinflamatorios, relajantes musculares, benzodiacepinas, precursores de catecolaminas y antagonistas betaadrenérgicos. Sin embargo, todavía no existe un medicamento definitivo de primera opción para ser utilizado de manera segura en los niños. Las benzodiacepinas todavía se usan en la práctica clínica, a pesar de la disminución en su indicación debido a efectos adversos como depresión y dependencia. Dentro de esta categoría, la Clonidina aparece en la literatura como una opción terapéutica, sin embargo, se asocia con varios efectos secundarios adversos. Por lo tanto, el benzodiacepina clonazepam aparece como una alternativa segura en comparación con la clonidina. ${ }^{12,18,19}$

Machado et al. (2011), ${ }^{20}$ considera que la placa oclusal es un tratamiento aceptable, pero como tratamiento farmacólogico el Clonazepam se destaca en el corto plazo, no generando dependencia. Además, señaló a través de una revisión sistemática que en cuanto a los tratamientos experimentales, la Clonidina y el dispositivo de avance mandibular son muy prometedores, sin embargo, pueden causar efectos secundarios adversos. 
Sin embargo otros estudios muestran que medicamentos como toxina botulínica tipo A, las benzodiacepinas, los anticonvulsivos, las betabloqueantes, la dopamina, los antidepressivos y los relajantes musculares, pueden ayudar a controlar el bruxismo, pero el uso de estos agentes en los niños está restringido, por lo que los fitoquímicos naturales a base de plantas se consideran alternativas viables para mejorar la calidad de vida del niño. $11,14,17,21,22$

Además de estas opciones, la evaluación del logopeda resultó ser importante, ya que permitió verificar el impacto en la motricidad orofacial debido al bruxismo así como las funciones alimentarias y respiratorias, lo que permite un tratamiento multidisciplinario..$^{5,22,23}$

Algunos estudios han podido verificar la efectividad de las intervenciones psicosociales, como el asesoramiento, los ejercicios de relajación, la hipnosis y la biorretroalimentación para disminuir la frecuencia e intensidad de esta condición. ${ }^{20}$ Vale la pena mencionar los efectos de los trastornos psiquiátricos en la infancia, ya que las personas afectadas por el trastorno por déficit de atención con hiperactividad (TDAH) tratados con medicamentos son más propensos a desarrollar bruxismo en comparación con las personas no afectadas. $^{12}$

Actualmente, la fisioterapia también se ha utilizado como una de las formas de tratar este trastorno, y los métodos más utilizados son: estimulación neuromuscular transcutánea y eléctrica por microcorriente, el ultrasonido, la crioterapia, la terapia infrarroja, el masaje terapéutico, la kinesioterapia, la acupuntura y la terapia con láser de bajo nivel.
El tratamiento realizado con láser de baja potencia es eficaz para reducir los síntomas derivados del bruxismo, como el dolor orofacial, la hinchazón y la hiperemia. Sin embargo, faltan estudios que demuestren su efectividad. ${ }^{7}$

Según la asociación brasileña de odontología central del bruxismo está relacionada con el movimiento mandibular involuntario, con eso, la analogía de que el intercambio de dentición estaría relacionado con el posible desarrollo del bruxismo se vuelve insostenible. Debido a que su origen está en el sistema nervioso central, el uso de aparatos ortodóncicos u ortopédicos no promueven cambios morfológicos. ${ }^{24}$

La limitaciones de este estudio se refieren a la muestra, ya que solo se incluyeron artículos disponibles en línea de forma gratuita y en inglés y portugués. Por lo tanto, se reconoce la posibilidad de que no se hayan incorporado todos los enfoques posibles al problema de investigación. Considerando que el tratamiento del bruxismo infantil tiende a ser multidisciplinario debido a los aspectos conductuales que involucran al niño, es necesario que los diferentes tipos de estudios incoporen aspectos asociados con la odontología, la medicina, la psicología y otras áreas en la ruta metodológica. ${ }^{5,11}$

\section{Conclusión}

Dado lo anterior, se concluyó que debido a que el bruxismo tiene una etiología amplia, su tratamiento tiende a ser un desafío para los profesionales de la salud. Por lo tanto, es necesario adoptar medidas basadas en terapias paliativas y la reducción de complicaciones 
patológicas, con el objetivo, sobre todo, de mejorar la calidad de vida de las personas. Además, a pesar de los avances en la investigación, todavía no hay consenso sobre un tratamiento específico y la mayoría no tiene una base científica suficiente para controlar/curar este hábito prejudicial.

\section{Referencias bibliográficas}

1. Feitosa GMA, Felix RCL, Sampaio DC, Vieira-Andrade RG, Santos CCO, Fonseca-Filho T. Bruxismo da infância: Perfil de comportamento, característica do sono e sintomatologia. Revista Bahiana de Odontologia, 2016 Jun; 7(2):94-104.

2. Oliveira ALBM, Fragelli C, Andrade MF. Abordagem multidisciplinar no tratamento do bruxismo infantil. Revista Uningá, 2017 nov; 25(1):1-8.

3. Restrepo CC, Medina I, Patiño I. Effect of occlusal splints on the temporomandibular disorders, dental wear and anxiety of bruxist children. European Journal Dentistry, 2011; 5 (4): 441-450.

4. Silva NR. Bruxismo: etiologia e tratamento. Revista Brasileira de Odontologia, Rio de Janeiro, 2019; 66(2):223-227.

5. Simões-Zenari M, Bitar ML. Fatores associados ao bruxismo em crianças de 4 a 6 anos. Pró-Fono Revista de Atualização Científica. 2010 out-dez; 22(4):465-472.

6. Cabral LC, Lopes AJC, Moura MB, Silva RR, Neto AJF, Júnior PCS. Bruxismo na infância: fatores e possíveis fatores de risco. Faculdade de Odontologia Lins/Unimep, 2018 jan-jun, 28(1): 41-51.

7. Salgueiro MDCC, Bortoletto CC, Horliana ACR, Mota ACC, Motta LJ, Motta PB et. Al. Evaluation of muscle activity, bite force and salivary cortisol in children with bruxism before and after low level laser applied to acupoints: study protocol for a randomised controlled trial. BMC Complementary and Alternative Medicine, 2017 Aug; 17(1):391-398.

8. Ribeiro TA, Freitas FCN. Bruxismo do sono na infância. Cadernos de odontologia da UNIFESO, 2019, 1(1):101-109.

9. Souza MT, Silva MD, Carvalho R. Revisão integrativa: o que é e como fazer? Einstein, 2010; 8(1): 102-106.

10. Bortoletto CC, Cordeiro da SF, Silva PF, Leal de Godoy CH, Albertini R, Motta LJ et. al. Evaluation of Cranio-cervical Posture in Children with Bruxism Before and After Bite Plate Therapy: A Pilot Project. Journal of Physical Therapy Science, 2014 Jul; 26(7):1125-1128.

11. Diniz MB, Silva RC, Zuanon ACC. Bruxismo na infância: um sinal de alerta para odontopediatras e pediatras. Revista Paulista de Pediatria [Internet], 2009 Sep [cited 2019 Oct 16]; 27(3): 329-334.

12. Machado E, Dal-Fabbro C, Cunali PA, Kaizer OB. Prevalence of sleep bruxism in children: A systematic review. Dental Press Journal of Orthodontics, 2014; 19(6): 54-61.

13. Ferreira-Bacci Adriana do Vale, Cardoso Carmen Lúcia Cardoso, Díaz-Serrano Kranya Victoria. Behavioral problems and emotional stress in children with bruxism. Brazilian Dental Journal, 2012; 23(3): 246-251.

14. Rahmati M, Moayedi A, Zakery Shahvari S, Golmirzaei J, Zahirinea M, Abbasi B. The effectof hydroxyzine on treating bruxismof 2- to 14-year-old children admitted to the clinic of Bandar Abbas Children Hospital in 2013-2014. Journal of Medicine and Life, 2015; 8(4): 241-244.

15. Alfaya A, Tannure PN, Barcelos R, Dip EC, Uemoto L, Gouvêa CVD. Clinical management of childhood bruxism. Revista Gaúcha de Odontologia, 2015; 63 (2): 207-212.

16. Silva CT, Primo LG, Mangabeira A, Maia LC, Fonseca-Gonçalves A. Homeopathic therapy for sleep bruxism in a child: Findings of a 2-year case report. Journal of Indian Society of Pedodontics and Preventive Dentistry, 2017; 35(4):381-3.

17. Lerardo G, Mazur M, Luzzi V, Calcagnile F, Ottolenghi L, Polimeni A. Treatments of sleep bruxism in children: A systematic review and meta-analysis. The Journal of Craniomandibular \& Sleep Practice. 2018, 38(2): 1-7.

18. Mostafavi SN, Jafari A, Hoseini SG, Khademian M, Kelishadi R. The efficacy of low and moderate dosage of diazepam on sleep bruxism in children: A randomized placebo-controlled clinical trial. Journal of Research in Medical Sciences. 2019; 24(8):1-6. 
19. Bortoletto CC, Cordeiro da Silva F, Salgueiro M da C, Motta LJ, Curiki LM, Mesquita-Ferarri RA et. Al. Evaluation of electromyographic signals in children with bruxism before and after therapy with Melissa Officinalis L-a randomized controlled clinical trial. Journal of Physical Therapy Science. 2016 Mar;28(3):738-742.

20. Machado E, Machado P, Cunali PA, Dal FC. Bruxismo do sono: possibilidades terapêuticas baseadas em evidências. Dental Press Journal of Orthodontics. [Internet]. 2011; 16(2): 58-64.

21. Yüce M, Karabekiroğlu K, Say GN, Müjdeci M, Oran M. Buspirone Use in the Treatment of AtomoxetineInduced Bruxism. Journal of Child and Adolescent Psychopharmacology, 2013 Nov; 23(9): 634-635.

22. Ghanizadeh A. Treatment of bruxism with hydroxyzine. European Review for Medical Pharmacological Sciences, 2013;17(6):839-841.

23. Ortu E, Pietropaoli D, Marchetti E, Marchili N, Marzo G, Monaco A. Bruxism in children: Use of the Functional Plane of Monaco (FPM). European Journal of Paediatric Dentistry. 2018; 19(4): 287-294.

24. Ortega AL. Nota de Esclarecimento: Bruxismo na Infância. Associação Brasileira de Odontopediatria (ABOPED), 2017. Acessado (2019 novembro 2) Disponível em: https://abodontopediatria.org.br/ site/?p=995. 